\title{
Characterization of the Strong Metric Subregularity of the Mordukhovich Subdifferential on Asplund Spaces
}

\author{
J. J. Wang ${ }^{1,2}$ and W. Song ${ }^{1,3}$ \\ ${ }^{1}$ Department of Mathematics, Harbin Institute of Technology, Harbin 150001, China \\ ${ }^{2}$ College of Computer Science and Information Engineering, Harbin Normal University, Harbin 150025, China \\ ${ }^{3}$ School of Mathematical Sciences, Harbin Normal University, Harbin 150025, China
}

Correspondence should be addressed to W. Song; ws_hrbnu@163.com

Received 26 March 2014; Accepted 11 June 2014; Published 1 July 2014

Academic Editor: Chong Li

Copyright (C) 2014 J. J. Wang and W. Song. This is an open access article distributed under the Creative Commons Attribution License, which permits unrestricted use, distribution, and reproduction in any medium, provided the original work is properly cited.

We mainly present several equivalent characterizations of the strong metric subregularity of the Mordukhovich subdifferential for an extended-real-valued lower semicontinuous, prox-regular, and subdifferentially continuous function acting on an Asplund space.

\section{Introduction}

This work is devoted to characterizations of the strong metric subregularity of the Mordukhovich subdifferential for an extended-real-valued prox-regular subdifferentially continuous function $f$ defined on an Asplund space.

Aragón and Geoffroy [1] have established remarkable equivalences between various metric regularity properties (including the (strong) metric subregularity and the (strong) metric regularity) of the subgradient mapping $\partial f$ and a local quadratic growth condition for a proper convex lower semicontinuous function $f$ defined on a Hilbert space $X$. Later, Drusvyatskiy and Lewis [2] proved that the characterization established in [1] for the strong metric regularity remains valid for the Mordukhovich subdifferential at $\bar{x}$ for 0 of a lower semicontinuous (not necessarily convex) function defined on $\mathbb{R}^{n}$ when the function is subdifferentially continuous at $\bar{x}$ for 0 . Subsequently, Mordukhovich and Nghia [3] generalized this result to Asplund spaces without the assumption of subdifferential continuity [3, Corollary 3.3]

Recently, Aragón and Geoffroy [4] established the equivalence between the strong metric subregularity of the subgradient mapping $\partial f$ for a proper lower semicontinuous (l.s.c.) convex function $f$ defined on a Banach space $X$ and the following quadratic growth condition:

$$
f(x) \geq f(\bar{x})+\left\langle\bar{x}^{*}, x-\bar{x}\right\rangle+\frac{c}{2}\|x-\bar{x}\|^{2} \quad \forall x \in U,
$$

where $U$ is a neighborhood of $\bar{x}$ and $c \in(0,1 / 2 \kappa)$. They raised the question whether the bound $c \in(0,1 / 2 \kappa)$ can be improved. Drusvyatskiy et al. [5] extended the above equivalence to the case, where the function $f$ is a proper lower semicontinuous function defined on an Asplund space and gave the affirmative answer to the above question via showing that the constant $c$ in (1) may be chosen arbitrarily in $(0,1 / \kappa)$.

In the present paper, motivated by the above results, we present several characterizations of the strong metric subregularity of the Mordukhovich subdifferential for an extended-real-valued l.s.c. prox-regular and subdifferentially continuous function acting on an Asplund space.

Section 2 contains necessary definitions and facts. In Section 3 we extend the known equivalences for strong metric subregularity of subdifferentials for convex functions to the class of prox-regular and subdifferentially continuous functions defined on the Asplund spaces (Theorem 12). Theorem 15 is concerning the relationship between the strong metric subregularity and contingent derivative of the Mordukhovich subdifferential.

\section{Preliminaries}

Throughout this paper, $X$ and $Y$ are Asplund spaces, that is, Banach spaces such that every separable subspace has a separable dual, and $X^{*}$ is the dual space of $X$. The symbol $\rightarrow$ always denotes the convergence relative to the norm while 
the symbol $\stackrel{w^{*}}{\longrightarrow}$ signifies the weak ${ }^{*}$ convergence in the dual space $X^{*}$. The closed ball centered at $x \in X$ of radius $r$ is denoted by $\mathbb{B}_{r}(x)$ while the closed unit and dual unit balls are denoted by $\mathbb{B}$ and $\mathbb{B}^{*}$, respectively. The distance function associated with a nonempty set $\Omega \in X$ is defined by

$$
d(x ; \Omega):=\inf _{y \in \Omega}\|x-y\|, \quad x \in X .
$$

Let $F: X \rightrightarrows Y$ be a set-valued mapping between Banach spaces $X$ and $Y$. The domain and graph are defined by

$$
\begin{gathered}
\operatorname{dom} F:=\{x \in X \mid F(x) \neq \emptyset\}, \\
\operatorname{gph} F:=\{(x, y) \in X \times Y \mid y \in F(x)\},
\end{gathered}
$$

respectively. For a mapping $F: X \rightrightarrows X^{*}$ between a Banach space $X$ and its dual $X^{*}$ we define the sequential PainlevéKuratowski outer limit by

$$
\begin{aligned}
& \underset{x \rightarrow \bar{x}}{\operatorname{Lim} \sup } F(x) \\
& :=\left\{x^{*} \in X^{*} \mid \exists x_{k} \longrightarrow \bar{x},\right. \\
& \left.x_{k}^{*} \stackrel{w^{*}}{\longrightarrow} x^{*} \text { with } x_{k}^{*} \in F\left(x_{k}\right) \forall k\right\} .
\end{aligned}
$$

Definition 1. Given a subset $K \subset X$ and a point $x \in K$, the contingent cone of $K$ at $x$ is defined by

$$
\begin{aligned}
T_{K}(x) & =\underset{\tau \downarrow 0}{\operatorname{Lim} \sup } \frac{K-x}{\tau} \\
& =\left\{v \mid \exists \tau_{k} \longrightarrow 0, \exists K \ni x_{k} \longrightarrow x, \text { s.t. } \frac{x_{k}-x}{\tau_{k}} \longrightarrow v\right\} .
\end{aligned}
$$

In stability analysis, the concept of metric regularity and its variants plays an important role; for more details and further references see, for example, [6-8]. Our study is focused on two key notions: metric subregularity and strong metric subregularity. They are defined as follows.

Definition 2. A mapping $F: X \rightrightarrows Y$ is said to be metrically subregular at $\bar{x}$ for $\bar{y}$ if $\bar{y} \in F(\bar{x})$ and there is a positive constant $\kappa$ along with neighborhoods $U$ of $\bar{x}$ and $V$ of $\bar{y}$ such that

$$
d\left(x ; F^{-1}(\bar{y})\right) \leq \kappa d(\bar{y} ; F(x) \cap V), \quad \forall x \in U
$$

Definition 3. A mapping $F: X \rightrightarrows Y$ is said to be strongly (metrically) subregular at $\bar{x}$ for $\bar{y}$ if $\bar{y} \in F(\bar{x})$ and there is a positive constant $\kappa$ along with neighborhoods $U$ of $\bar{x}$ and $V$ of $\bar{y}$ such that

$$
\|x-\bar{x}\| \leq \kappa d(\bar{y} ; F(x) \cap V), \quad \forall x \in U .
$$

We notice that the strong metric subregularity of $F$ at $\bar{x}$ for $\bar{y}$ is equivalent to the metric subregularity if $\bar{x}$ is an isolated point of $F^{-1}(\bar{y})$. The definition of metric subregularity can be simplified in the following way:

$$
d\left(x ; F^{-1}(\bar{y})\right) \leq \kappa d(\bar{y} ; F(x)), \quad \forall x \in U^{\prime} .
$$

For a possibly smaller neighborhood $U^{\prime}$ of $\bar{x}$, see [1] for details. Likewise, the definition of strong subregularity can be simplified as

$$
\|x-\bar{x}\| \leq \kappa d(\bar{y} ; F(x)), \quad \forall x \in U^{\prime} .
$$

For an extended-real-valued function $f: X \rightarrow \mathbb{\mathbb { R }}:=$ $(-\infty ;+\infty]$ we define the domain of $f$ to be

$$
\operatorname{dom} f:=\{x \in X \mid f(x)<+\infty\} .
$$

In this paper, we assume that all the extended-real-valued functions are proper, that is, not identically equal to $+\infty$, and lower semicontinuous (l.s.c.) on $X$.

The fundamental tools for studying general nonsmooth function are subdifferentials. The following two subdifferential notions are used in this paper.

Definition 4. Consider $f: X \rightarrow \overline{\mathbb{R}}$ and $x \in \operatorname{dom} f$.

(i) $p \in X^{*}$ is a proximal subgradient if there is $r \geq 0$ with

$$
f\left(x^{\prime}\right) \geq f(x)+\left\langle p, x^{\prime}-x\right\rangle-\frac{r}{2}\left\|x^{\prime}-x\right\|^{2}
$$

for any $x^{\prime}$ from some neighborhood of $x$. The proximal subdifferential $\partial_{p} f(x)$ of $f$ at $x$ is the collection of all proximal subgradients of $f$ at $x$.

(ii) The (basic, limiting, and Mordukhovich) subdifferential of $f$ at $x$ is

$$
\partial f(x):=\limsup _{x^{\prime} \stackrel{f}{\rightarrow} x} \partial_{p} f(x),
$$

where the symbol $x^{\prime} \stackrel{f}{\rightarrow} x$ means that $x^{\prime} \rightarrow x$ with $f\left(x^{\prime}\right) \rightarrow f(x)$.

When $f$ is convex, $\partial f(x)$ is the usual subgradient set of convex analysis. When $f$ is smooth, $\partial f(x)$ is the singleton $\nabla f(x)$.

Definition 5. Let $f: X \rightarrow \overline{\mathbb{R}}$ and $\bar{x} \in \operatorname{dom} f$. We say that $f$ is prox-regular at $\bar{x}$ for $\bar{v} \in \partial f(\bar{x})$ if there exist $\varepsilon>0$ and $r \geq 0$ such that

$$
f\left(x^{\prime}\right) \geq f(x)+\left\langle v, x^{\prime}-x\right\rangle-\frac{r}{2}\left\|x^{\prime}-x\right\|^{2} \quad \forall x^{\prime} \in \mathbb{B}_{\varepsilon}(\bar{x}),
$$

whenever $x \in \mathbb{B}_{\varepsilon}(\bar{x}),|f(x)-f(\bar{x})|<\varepsilon$, and $v \in \partial f(x)$ with $\|v-\bar{v}\|<\varepsilon$. If this holds for every $\bar{v} \in \partial f(\bar{x})$, we say that $f$ is prox-regular at $\bar{x}$.

Definition 6. A function $f: X \rightarrow \overline{\mathbb{R}}$ is subdifferentially continuous at $\bar{x} \in \operatorname{dom} f$ for $\bar{v} \in \partial f(\bar{x})$ if for every $\varepsilon>0$ there exists $\delta>0$ such that $|f(x)-f(\bar{x})|<\varepsilon$ whenever $x \in \mathbb{B}_{\delta}(\bar{x})$ and $\|v-\bar{v}\|<\delta$ with some $v \in \partial f(x)$. If this occurs for all $\bar{v} \in \partial f(\bar{x})$, we say that $f$ is subdifferentially continuous at $\bar{x}$. 
The concepts of prox-regularity and subdifferential continuity were introduced in [9] and were further studied in $[10,11]$. It was shown in those works that the class of functions with these properties is quite large. It includes $\mathscr{C}^{2}$ functions, l.s.c. proper convex functions, and many functions that typically might be encountered in finite-dimensional optimization.

The well-known Ekeland's variational principle [12] plays an important role in this paper.

Theorem 7 (Ekeland's variational principle). Let $(X, d)$ be a complete metric space and $f: X \rightarrow(-\infty,+\infty]$ a proper lower semicontinuous function bounded from below. Suppose that, for some $u \in X$ and some $\varepsilon>0$,

$$
f(u) \leq \inf _{x \in X} f(x)+\varepsilon .
$$

Then, for every $\lambda>0$ there exists some point $v \in X$ such that

$$
\begin{gathered}
d(v, u) \leq \lambda, \\
f(v)+\frac{\varepsilon}{\lambda} d(v, u) \leq f(u), \\
f(x)>f(v)-\frac{\varepsilon}{\lambda} d(v, x), \quad \forall x \neq v .
\end{gathered}
$$

\section{Characterizing Strong Subregularity Properties of the Subdifferential}

In this section, we will give characterizations of the strong metric subregularity of the Mordukhovich subdifferential of extended-real-valued prox-regular subdifferentially continuous functions. Namely, we provide some equivalent conditions to the strong metric subregularity of subdifferentials of such functions including quadratic growth properties and locally strongly monotone of subdifferentials.

The following theorem is implied in [5, Corollary 3.3]; we present a slightly different proof.

Theorem 8. Let $f: X \rightarrow \overline{\mathbb{R}}$ and $\left(\bar{x}, \bar{x}^{*}\right) \in$ gph $\partial f$. Suppose that the subdifferential $\partial f$ is strongly metrically subregular at $\left(\bar{x}, \bar{x}^{*}\right)$ with modulus $\kappa>0$. Then, the following assertions are equivalent:

(i) there are real numbers $\alpha \in\left(0, \kappa^{-1}\right)$ and $\delta>0$ such that

$$
f(x) \geq f(\bar{x})+\left\langle\bar{x}^{*}, x-\bar{x}\right\rangle+\frac{\alpha}{2}\|x-\bar{x}\|^{2} \quad \forall x \in \mathbb{B}_{\delta}(\bar{x}),
$$

(ii) for any real number $\bar{\alpha} \in\left(0, \kappa^{-1}\right)$, there is a real number $\eta>0$ such that

$$
f(x) \geq f(\bar{x})+\left\langle\bar{x}^{*}, x-\bar{x}\right\rangle+\frac{\bar{\alpha}}{2}\|x-\bar{x}\|^{2} \quad \forall x \in \mathbb{B}_{\eta}(\bar{x}) .
$$

Proof. Implication (ii) $\Rightarrow$ (i) holds trivially.

Next, we focus on (i) $\Rightarrow$ (ii). Since the subdifferential $\partial f$ is strongly metrically subregular at $\left(\bar{x}, \bar{x}^{*}\right)$ with modulus $\kappa>0$, there is some positive constant $a$ such that

$$
\|x-\bar{x}\| \leq \kappa d\left(\bar{x}^{*} ; \partial f(x)\right) \quad \forall x \in \operatorname{int} \mathbb{B}_{a}(\bar{x}) .
$$

Let $\theta=\min \{a, \delta\}$.

We start by showing that

$$
f(x) \geq f(\bar{x})+\left\langle\bar{x}^{*}, x-\bar{x}\right\rangle+\frac{1+\alpha \kappa}{4 \kappa}\|x-\bar{x}\|^{2}
$$

for all $x \in \mathbb{B}_{\lambda \theta}(\bar{x})$, where $\lambda \in(1 / 2,2 / 3)$. Suppose that (19) is not true and there is some $z \in \mathbb{B}_{\lambda \theta}(\bar{x})$ such that

$$
f(z)<f(\bar{x})+\left\langle\bar{x}^{*}, z-\bar{x}\right\rangle+\frac{1+\alpha \kappa}{4 \kappa}\|z-\bar{x}\|^{2} .
$$

This together with (16) implies that $z \neq \bar{x}$ and

$$
\begin{aligned}
& \inf _{x \in \mathbb{B}_{\theta}(\bar{x})}\left\{f(x)-\left\langle\bar{x}^{*}, x-\bar{x}\right\rangle-\frac{\alpha}{2}\|x-\bar{x}\|^{2}\right\} \\
& \quad \geq f(\bar{x}) \\
& >f(z)-\left\langle\bar{x}^{*}, z-\bar{x}\right\rangle-\frac{1+\alpha \kappa}{4 \kappa}\|z-\bar{x}\|^{2} \\
& \quad=f(z)-\left\langle\bar{x}^{*}, z-\bar{x}\right\rangle-\frac{\alpha}{2}\|z-\bar{x}\|^{2}-\frac{1-\alpha \kappa}{4 \kappa}\|z-\bar{x}\|^{2} .
\end{aligned}
$$

Hence, there is some $\varepsilon>0$ slightly smaller than

$$
\frac{1-\alpha \kappa}{4 \kappa}\|z-\bar{x}\|^{2}
$$

such that

$$
\begin{gathered}
\inf _{x \in \mathbb{B}_{\theta}(\bar{x})}\left\{f(x)-\left\langle\bar{x}^{*}, x-\bar{x}\right\rangle-\frac{\alpha}{2}\|x-\bar{x}\|^{2}\right\}+\varepsilon \\
>f(z)-\left\langle\bar{x}^{*}, z-\bar{x}\right\rangle-\frac{\alpha}{2}\|z-\bar{x}\|^{2} .
\end{gathered}
$$

Since $f$ is l.s.c. on $\mathbb{B}_{\theta}$, by Ekeland's variational principle (Theorem 7), there exists some $u \in \mathbb{B}_{\theta}(\bar{x})$ such that

$$
\|u-z\| \leq \frac{1}{2}\|z-\bar{x}\|
$$

and for all $x \in \mathbb{B}_{\theta}(\bar{x})$

$$
\begin{aligned}
& f(x)-\left\langle\bar{x}^{*}, x-\bar{x}\right\rangle-\frac{\alpha}{2}\|x-\bar{x}\|^{2}+\frac{2 \varepsilon}{\|z-\bar{x}\|}\|x-u\| \\
& \geq f(u)-\left\langle\bar{x}^{*}, u-\bar{x}\right\rangle-\frac{\alpha}{2}\|u-\bar{x}\|^{2} .
\end{aligned}
$$

This implies that $u$ minimizes the function

$$
f(x)-\left\langle\bar{x}^{*}, x-\bar{x}\right\rangle-\frac{\alpha}{2}\|x-\bar{x}\|^{2}+\frac{2 \varepsilon}{\|z-\bar{x}\|}\|x-u\|
$$


over $\mathbb{B}_{\theta}(\bar{x})$ and

$$
\|u-\bar{x}\| \leq \frac{3}{2}\|z-\bar{x}\|<\theta .
$$

Then, Fermat's stationary rule along with the subdifferential sum rule ([8, Theorem 3.6]) implies the inclusions

$$
\begin{aligned}
0 & \in \partial\left(f(\cdot)-\left\langle\bar{x}^{*}, \cdot-\bar{x}\right\rangle-\frac{\alpha}{2}\|\cdot-\bar{x}\|^{2}+\frac{2 \varepsilon}{\|z-\bar{x}\|}\|\cdot-u\|\right)(u) \\
& \subset \partial f(u)-\bar{x}^{*}+\left[\alpha\|u-\bar{x}\|+\frac{2 \varepsilon}{\|z-\bar{x}\|}\right] \mathbb{B}^{*} .
\end{aligned}
$$

Therefore,

$$
\begin{aligned}
d\left(\bar{x}^{*} ; \partial f(u)\right) & \leq \alpha\|u-\bar{x}\|+\frac{2 \varepsilon}{\|z-\bar{x}\|} \\
& <\alpha\|u-\bar{x}\|+\frac{1-\alpha \kappa}{2 \kappa}\|z-\bar{x}\| .
\end{aligned}
$$

Additionally, since

$$
\begin{aligned}
\|z-\bar{x}\| & \leq\|u-z\|+\|u-\bar{x}\| \\
& \leq \frac{1}{2}\|z-\bar{x}\|+\|u-\bar{x}\|,
\end{aligned}
$$

one has

$$
\|z-\bar{x}\| \leq 2\|u-\bar{x}\|
$$

and thus

$$
\begin{aligned}
d\left(\bar{x}^{*} ; \partial f(u)\right) & <\alpha\|u-\bar{x}\|+\frac{1-\alpha \kappa}{\kappa}\|u-\bar{x}\| \\
& =\frac{1}{\kappa}\|u-\bar{x}\| .
\end{aligned}
$$

This strict inequality contradicts $(18)$, since $u \in$ int $\mathbb{B}_{a}(\bar{x})$ by (27).

By induction we may then construct from (19) a strictly positive sequence $\left\{\alpha_{k}\right\}$ satisfying

$$
\begin{array}{r}
f(x) \geq f(\bar{x})+\left\langle\bar{x}^{*}, x-\bar{x}\right\rangle+\frac{\alpha_{k}}{2}\|x-\bar{x}\|^{2} \\
\forall x \in \mathbb{B}_{\theta(\lambda)^{k}}(\bar{x})
\end{array}
$$

with $\alpha_{k}:=\left(1+\alpha_{k-1} \kappa\right) / 2 \kappa \in\left(0, \kappa^{-1}\right)$ for $k \in \mathbb{N}$ and $\alpha_{0}:=\alpha$. Consequently it gives

$$
\alpha_{k}=\kappa^{-1}\left(1-\frac{1}{2^{k}}\right)+\frac{\alpha}{2^{k}} \longrightarrow \kappa^{-1} \quad \text { as } k \longrightarrow+\infty
$$

which completes the proof.

Corollary 9. Let $f: X \rightarrow \overline{\mathbb{R}}$ and $\left(\bar{x}, \bar{x}^{*}\right) \in$ gph $\partial f$. Suppose that the subdifferential $\partial f$ is strongly metrically subregular at $\left(\bar{x}, \bar{x}^{*}\right)$ with modulus $\kappa>0$. Then, the following assertions are equivalent: (i) there are real numbers $r \in\left(0, \kappa^{-1}\right)$ and $\delta>0$ such that $f(x) \geq f(\bar{x})+\left\langle\bar{x}^{*}, x-\bar{x}\right\rangle-\frac{r}{2}\|x-\bar{x}\|^{2} \quad \forall x \in \mathbb{B}_{\delta}(\bar{x})$,

(ii) for any real number $\bar{\alpha} \in\left(0, \kappa^{-1}\right)$, there is a real number $\eta>0$ such that

$$
f(x) \geq f(\bar{x})+\left\langle\bar{x}^{*}, x-\bar{x}\right\rangle+\frac{\bar{\alpha}}{2}\|x-\bar{x}\|^{2} \quad \forall x \in \mathbb{B}_{\eta}(\bar{x}) .
$$

Proof. By [5, Corollary 3.3] and Theorem 8, we have that the equivalence (i) $\Leftrightarrow$ (ii) holds.

The following theorem provides a characterization of strong metric subregularity of the subdifferential for a proxregular and subdifferentially continuous function.

Theorem 10. Given $f: X \rightarrow \overline{\mathbb{R}}$ and a pair $\left(\bar{x}, \bar{x}^{*}\right) \in$ gph $\partial f$, let $f$ be prox-regular at $\bar{x}$ for $\bar{x}^{*}$ with $r>0$ and subdifferentially continuous at $\bar{x}$ for $\bar{x}^{*}$. Then, $\partial f$ is strongly metrically subregular at $\left(\bar{x}, \bar{x}^{*}\right)$ with modulus $\kappa$ satisfying $\kappa^{-1}>r$ if and only if for any real number $\bar{\alpha} \in\left(0, \kappa^{-1}\right)$, there is a real number $\eta>0$ such that

$$
f(x) \geq f(\bar{x})+\left\langle\bar{x}^{*}, x-\bar{x}\right\rangle+\frac{\bar{\alpha}}{2}\|x-\bar{x}\|^{2} \quad \forall x \in \mathbb{B}_{\eta}(\bar{x}) .
$$

Proof. Suppose that $\partial f$ is strongly metrically subregular at $\left(\bar{x}, \bar{x}^{*}\right)$ with modulus $\kappa$ satisfying $\kappa^{-1}>r$. Since $f$ is proxregular at $\bar{x}$ for $\bar{x}^{*}$ with $r>0$ and subdifferentially continuous at $\bar{x}$ for $\bar{x}^{*}$, by Definitions 5 and 6 , there exists a real number $\varepsilon_{1}>0$ such that

$$
f(x) \geq f(\bar{x})+\left\langle\bar{x}^{*}, x-\bar{x}\right\rangle-\frac{r}{2}\|x-\bar{x}\|^{2} \quad \forall x \in \mathbb{B}_{\varepsilon_{1}}(\bar{x}) .
$$

Hence, by Corollary 9, we have that inequality (37) holds.

To justify the converse implication, assume that (37) holds for some $\kappa^{-1}>\bar{\alpha}>r$ and $\eta>0$. Since $f$ is prox-regular at $\bar{x}$ for $\bar{x}^{*}$ with $r \in\left(0, \kappa^{-1}\right)$ and subdifferentially continuous at $\bar{x}$ for $\bar{x}^{*}$, there is a constant $\varepsilon \in(0, \eta)$ such that

$$
f\left(x^{\prime}\right) \geq f(x)+\left\langle x^{*}, x^{\prime}-x\right\rangle-\frac{r}{2}\left\|x^{\prime}-x\right\|^{2}
$$

for all $x^{\prime}, x \in \mathbb{B}_{\varepsilon}(\bar{x}), x^{*} \in \partial f(x) \cap \mathbb{B}_{\varepsilon}\left(\bar{x}^{*}\right)$. Fix any point $x \in$ $\mathbb{B}_{\varepsilon}(\bar{x})$; if $d\left(\bar{x}^{*} ; \partial f(x)\right) \geq \varepsilon$, it clearly follows that $\|x-\bar{x}\| \leq$ $d\left(\bar{x}^{*} ; \partial f(x)\right)$. Hence, we may suppose that $d\left(\bar{x}^{*} ; \partial f(x)\right)<\varepsilon$; it follows from (37) and (39) that

$$
\begin{aligned}
& \left\|x^{*}-\bar{x}^{*}\right\|\|x-\bar{x}\| \\
& \geq\left\langle x^{*}-\bar{x}^{*}, x-\bar{x}\right\rangle=\left\langle x^{*}, x-\bar{x}\right\rangle-\left\langle\bar{x}^{*}, x-\bar{x}\right\rangle \\
& \geq f(x)-f(\bar{x})-\frac{r}{2}\|x-\bar{x}\|^{2}-\left\langle\bar{x}^{*}, x-\bar{x}\right\rangle \\
& =f(x)-f(\bar{x})-\left\langle\bar{x}^{*}, x-\bar{x}\right\rangle-\frac{r}{2}\|x-\bar{x}\|^{2} \\
& \geq \frac{\bar{\alpha}-r}{2}\|x-\bar{x}\|^{2},
\end{aligned}
$$


which implies that

$$
\begin{aligned}
& \left\|x^{*}-\bar{x}^{*}\right\| \\
& \quad \geq \frac{\bar{\alpha}-r}{2}\|x-\bar{x}\| \quad \text { whenever } x^{*} \in \partial f(x) \cap \mathbb{B}_{\varepsilon}\left(\bar{x}^{*}\right) .
\end{aligned}
$$

By $d\left(\bar{x}^{*} ; \partial f(x)\right)<\varepsilon$, the latter implies that $d\left(\bar{x}^{*} ; \partial f(x)\right) \geq$ $((\bar{\alpha}-r) / 2)\|x-\bar{x}\|$, which justifies the strong metric subregularity of $\partial f$ at $\left(\bar{x}, \bar{x}^{*}\right)$ and thus completes the proof of the theorem.

Definition 11 ([4, Definition 3.4]). Let $T: X \rightrightarrows X^{*}$. The point $\left(\bar{x}, \bar{x}^{*}\right) \in$ gph $T$ is locally strongly monotonically related to gph $T$ if there exist neighborhoods $U$ of $\bar{x}$ and $V$ of $\bar{x}^{*}$ together with a constant $c>0$ such that

$$
\begin{aligned}
& \left\langle x^{*}-\bar{x}^{*}, x-\bar{x}\right\rangle \\
& \quad \geq c\|x-\bar{x}\|^{2}, \quad \forall\left(x, x^{*}\right) \in \operatorname{gph} T \cap(U \times V) .
\end{aligned}
$$

Now we are going to characterize the strong metric subregularity of the Mordukhovich subdifferential for an extended-real-valued l.s.c. prox-regular and subdifferentially continuous function acting on an Asplund space. Let us note that the equivalences of statements (i)-(iv) have been already established in [4, Theorem 3.6] in the convex setting. We show the equivalences of statements (i)-(iv) in nonconvex case, and we prove that they are also equivalent to statement $(\mathrm{v})$.

Theorem 12 (strong metric subregularity of the subdifferential). Let $f: X \rightarrow \overline{\mathbb{R}}$ and $\left(\bar{x}, \bar{x}^{*}\right) \in$ gph $\partial f$. If $f$ is proxregular at $\bar{x}$ for $\bar{x}^{*}$ with $r>0$ and subdifferentially continuous at $\bar{x}$ for $\bar{x}^{*}$, then the following statements are equivalent:

(i) the subdifferential $\partial f$ is strongly metrically subregular at $\left(\bar{x}, \bar{x}^{*}\right)$ with modulus $\kappa$ satisfying $\kappa^{-1}>r$,

(ii) for any real number $\bar{\alpha} \in\left(0, \kappa^{-1}\right)$, there is an $\eta>0$ such that

$$
\begin{array}{r}
f(x) \geq f(\bar{x})+\left\langle\bar{x}^{*}, x-\bar{x}\right\rangle+\frac{\bar{\alpha}}{2}\|x-\bar{x}\|^{2} \\
\forall x \in \mathbb{B}_{\eta}(\bar{x}),
\end{array}
$$

(iii) there exist neighborhoods $U$ of $\bar{x}$ and $V$ of $\bar{x}^{*}$ and $c>0$ such that

$$
\begin{array}{r}
\left\langle x^{*}-\bar{x}^{*}, x-\bar{x}\right\rangle \geq c\|x-\bar{x}\|^{2}, \\
\forall x \in U, \quad x^{*} \in \partial f(x) \cap V,
\end{array}
$$

(iv) the point $\left(\bar{x}, \bar{x}^{*}\right)$ is locally strongly monotonically related to gph $\partial f$,

(v) for any $\bar{\alpha} \in\left(0, \kappa^{-1}\right)$, there is a neighborhood $U$ of $\bar{x}$ such that, for all $x \in U$ and $\lambda \in(0,1)$,

$$
\begin{aligned}
& f((1-\lambda) x+\lambda \bar{x}) \\
& \quad \leq(1-\lambda) f(x)+\lambda f(\bar{x})-\frac{\bar{\alpha}}{2} \lambda(1-\lambda)\|x-\bar{x}\|^{2} .
\end{aligned}
$$

Proof. The equivalence (i) $\Leftrightarrow$ (ii) has been already proved in Theorem 10.

To show the implication (ii) $\Rightarrow$ (iii) suppose that (ii) holds for some $\kappa^{-1}>\bar{\alpha}>r$ and $\eta>0$. Since $f$ is prox-regular at $\bar{x}$ for $\bar{x}^{*}$ with $r \in\left(0, \kappa^{-1}\right)$ and subdifferentially continuous at $\bar{x}$ for $\bar{x}^{*}$, there is an $\varepsilon \in(0, \delta)$ such that

$$
f\left(x^{\prime}\right) \geq f(x)+\left\langle x^{*}, x^{\prime}-x\right\rangle-\frac{r}{2}\left\|x^{\prime}-x\right\|^{2}
$$

for all $x^{\prime}, x \in \mathbb{B}_{\mathcal{\varepsilon}}(\bar{x}), x^{*} \in \partial f(x) \cap \mathbb{B}_{\mathcal{\varepsilon}}\left(\bar{x}^{*}\right)$.

By letting $x^{\prime}$ equal $\bar{x}$, we obtain

$$
\begin{gathered}
\left\langle x^{*}, x-\bar{x}\right\rangle+\frac{r}{2}\|x-\bar{x}\|^{2} \geq f(x)-f(\bar{x}) \\
\forall x \in \mathbb{B}_{\varepsilon}(\bar{x}), \quad x^{*} \in \partial f(x) \cap \mathbb{B}_{\varepsilon}\left(\bar{x}^{*}\right) .
\end{gathered}
$$

It follows from (43) that

$$
f(x)-f(\bar{x}) \geq\left\langle\bar{x}^{*}, x-\bar{x}\right\rangle+\frac{\bar{\alpha}}{2}\|x-\bar{x}\|^{2} \quad \forall x \in \mathbb{B}_{\varepsilon}(\bar{x}) .
$$

Thus,

$$
\begin{aligned}
\left\langle x^{*}-\bar{x}^{*}, x-\bar{x}\right\rangle & =\left\langle x^{*}, x-\bar{x}\right\rangle-\left\langle\bar{x}^{*}, x-\bar{x}\right\rangle \\
& \geq \frac{\bar{\alpha}-r}{2}\|x-\bar{x}\|^{2},
\end{aligned}
$$

and (iii) holds with $c=(\bar{\alpha}-r) / 2$.

The implication (iii) $\Rightarrow$ (iv) is straightforward.

To show the implication (iv) $\Rightarrow$ (i) let us assume that (iv) holds. There are some neighborhoods $U$ of $\bar{x}$ and $V$ of $\bar{x}^{*}$ and a positive $c$ such that

$$
\begin{aligned}
& \left\langle x^{*}-\bar{x}^{*}, x-\bar{x}\right\rangle \\
& \quad \geq c\|x-\bar{x}\|^{2}, \quad \forall\left(x, x^{*}\right) \in \operatorname{gph} \partial f \cap(U \times V) .
\end{aligned}
$$

Pick any $x \in U \backslash\{\bar{x}\}$; if $\partial f(x) \cap V=\emptyset$ we are done. Otherwise, for any $x^{*} \in \partial f(x) \cap V$, we have

$$
\|x-\bar{x}\|^{2} \leq \frac{1}{c}\left\langle x^{*}-\bar{x}^{*}, x-\bar{x}\right\rangle \leq \frac{1}{c}\left\|x^{*}-\bar{x}^{*}\right\|\|x-\bar{x}\|,
$$

which implies that

$$
\|x-\bar{x}\| \leq \frac{1}{c} d\left(\bar{x}^{*} ; \partial f(x) \cap V\right)
$$

that is, the subdifferential $\partial f$ is strongly metrically subregular at $\left(\bar{x}, \bar{x}^{*}\right)$.

Finally, we prove the equivalence (ii) $\Leftrightarrow(\mathrm{v})$. Suppose that (v) holds. Let $x \in U, \lambda \in(0,1)$, and $\bar{\alpha} \in\left(0, \kappa^{-1}\right)$. From (45) we get

$$
\begin{aligned}
& (1-\lambda) f(x) \\
& \quad \geq f((1-\lambda) x+\lambda \bar{x})-\lambda f(\bar{x})+\frac{\bar{\alpha}}{2} \lambda(1-\lambda)\|x-\bar{x}\|^{2}
\end{aligned}
$$


that is,

$$
\begin{aligned}
f(x) \geq & \frac{1}{1-\lambda} f((1-\lambda) x+\lambda \bar{x}) \\
& -\frac{\lambda}{1-\lambda} f(\bar{x})+\frac{\bar{\alpha}}{2} \lambda\|x-\bar{x}\|^{2} .
\end{aligned}
$$

Moreover, by (46),

$$
\begin{aligned}
& f((1-\lambda) x+\lambda \bar{x}) \\
& \quad \geq f(\bar{x})+(1-\lambda)\left\langle\bar{x}^{*}, x-\bar{x}\right\rangle-\frac{r}{2}(1-\lambda)^{2}\|x-\bar{x}\|^{2},
\end{aligned}
$$

which together with inequality (54) gives

$$
\begin{aligned}
f(x) \geq & f(\bar{x})+\left\langle\bar{x}^{*}, x-\bar{x}\right\rangle+\frac{\bar{\alpha}}{2} \lambda\|x-\bar{x}\|^{2} \\
& -\frac{r}{2}(1-\lambda)\|x-\bar{x}\|^{2} .
\end{aligned}
$$

Letting $\lambda \uparrow 1$ in this inequality, we obtain (43).

Suppose now that (ii) holds. Let $x \in$ int $\mathbb{B}_{\eta}(\bar{x})$ and $\lambda \epsilon$ $(0,1)$. Then, (56) holds. This together with (55) implies (45) which completes the proof.

Let us note that, by Fermat's stationary rule, if a point $\bar{x}$ is a local minimizer of the function $f$, then we have $0 \in \partial f(\bar{x})$. By Theorem $12, \partial f$ is strongly metrically subregular at $\bar{x}$ for 0 with modulus $\kappa>0$ if and only if $f$ satisfies the following quadratic growth condition:

$$
f(x) \geq \inf f+c\|x-\bar{x}\|^{2} \quad \forall x \in \mathbb{B}_{\delta}(\bar{x}),
$$

where $c$ is a positive number.

The following result on the strong subregularity of the subdifferential of the sum of two functions is a straightforward consequence of Theorem 12.

Corollary 13. Let $f, g: X \rightarrow \overline{\mathbb{R}},\left(\bar{x}, \bar{x}^{*}\right) \in$ gph $\partial f$, and $\left(\bar{x}, \bar{y}^{*}\right) \in$ gph $\partial g$. Assume that $f$ is prox-regular at $\bar{x}$ for $\bar{x}^{*}$ with the constant $r_{1}$ and subdifferentially continuous at $\bar{x}$ for $\bar{x}^{*}$. Assume that $g$ is prox-regular at $\bar{x}$ for $\bar{y}^{*}$ with the constant $r_{2}$ and subdifferentially continuous at $\bar{x}$ for $\bar{y}^{*}$. If $\partial f$ is strongly metrically subregular at $\left(\bar{x}, \bar{x}^{*}\right)$ with modulus $\kappa_{1}, \kappa_{1}^{-1}>r_{1}$ and $\partial g$ is strongly metrically subregular at $\left(\bar{x}, \bar{y}^{*}\right)$ with modulus $\kappa_{2}$, $\kappa_{2}^{-1}>r_{2}$, then $\partial(f+g)$ is strongly metrically subregular at $\bar{x}$ for $\bar{x}^{*}+\bar{y}^{*}$.

Proof. By Theorem 12, the strong metrical subregularity of $\partial f$ at $\left(\bar{x}, \bar{x}^{*}\right)$ with modulus $\kappa_{1}$ yields, for any $\kappa_{1}^{-1}>\bar{\alpha}_{1}>r_{1}$, the existence of a real number $\delta_{1}>0$ such that

$$
f(x) \geq f(\bar{x})+\left\langle\bar{x}^{*}, x-\bar{x}\right\rangle+\frac{\bar{\alpha}_{1}}{2}\|x-\bar{x}\|^{2} \quad \forall x \in \mathbb{B}_{\delta_{1}}(\bar{x}) .
$$

The strong metrical subregularity of $\partial g$ at $\left(\bar{x}, \bar{y}^{*}\right)$ with modulus $\kappa_{2}$ implies that, for any $\kappa_{2}^{-1}>\bar{\alpha}_{2}>r_{2}$, the existence of a real number $\delta_{2}>0$ such that

$$
g(x) \geq g(\bar{x})+\left\langle\bar{y}^{*}, x-\bar{x}\right\rangle+\frac{\bar{\alpha}_{2}}{2}\|x-\bar{x}\|^{2} \quad \forall x \in \mathbb{B}_{\delta_{2}}(\bar{x}) .
$$

It follows from (58) and (59) that

$$
\begin{aligned}
(f+g)(x) \geq & (f+g)(\bar{x})+\left\langle\bar{x}^{*}+\bar{y}^{*}, x-\bar{x}\right\rangle \\
& +\frac{\bar{\alpha}_{1}+\bar{\alpha}_{2}}{2}\|x-\bar{x}\|^{2} \quad \forall x \in \mathbb{B}_{\delta}(\bar{x}),
\end{aligned}
$$

where $\delta=\min \left\{\delta_{1}, \delta_{2}\right\}$.

Since $f$ is prox-regular at $\bar{x}$ for $\bar{x}^{*}$ with $r_{1} \in\left(0, \kappa_{1}^{-1}\right)$ and subdifferentially continuous at $\bar{x}$ for $\bar{x}^{*}$ and $g$ is prox-regular at $\bar{x}$ for $\bar{y}^{*}$ with $r_{2} \in\left(0, \kappa_{2}^{-1}\right)$ and subdifferentially continuous at $\bar{x}$ for $\bar{y}^{*}$, there is a constant $\varepsilon \in(0, \delta)$ such that

$$
\begin{aligned}
& f(\bar{x}) \geq f(x)+\left\langle x^{*}, \bar{x}-x\right\rangle-\frac{r_{1}}{2}\|\bar{x}-x\|^{2}, \\
& g(\bar{x}) \geq g(x)+\left\langle y^{*}, \bar{x}-x\right\rangle-\frac{r_{2}}{2}\|\bar{x}-x\|^{2}
\end{aligned}
$$

for all $x \in \mathbb{B}_{\varepsilon}(\bar{x}), x^{*} \in \partial f(x) \cap \mathbb{B}_{\varepsilon}\left(\bar{x}^{*}\right), y^{*} \in \partial g(x) \cap \mathbb{B}_{\varepsilon}\left(\bar{y}^{*}\right)$. It follows from (61) and (62) that

$$
\begin{aligned}
& (f+g)(\bar{x}) \\
& \quad \geq(f+g)(x)+\left\langle x^{*}+y^{*}, \bar{x}-x\right\rangle-\frac{r_{1}+r_{2}}{2}\|x-\bar{x}\|^{2}
\end{aligned}
$$

for all $x \in \mathbb{B}_{\varepsilon}(\bar{x}), x^{*} \in \partial f(x) \cap \mathbb{B}_{\varepsilon}\left(\bar{x}^{*}\right), y^{*} \in \partial g(x) \cap \mathbb{B}_{\varepsilon}\left(\bar{y}^{*}\right)$. Inequality (60) together with (63) gives

$$
\begin{aligned}
& \left\langle\left(x^{*}+y^{*}\right)-\left(\bar{x}^{*}+\bar{y}^{*}\right), x-\bar{x}\right\rangle \\
& \quad \geq \frac{\left(\bar{\alpha}_{1}-r_{1}\right)+\left(\bar{\alpha}_{2}-r_{2}\right)}{2}\|x-\bar{x}\|^{2}
\end{aligned}
$$

for all $x \in \mathbb{B}_{\varepsilon}(\bar{x}), x^{*} \in \partial f(x) \cap \mathbb{B}_{\varepsilon}\left(\bar{x}^{*}\right), y^{*} \in \partial g(x) \cap \mathbb{B}_{\varepsilon}\left(\bar{y}^{*}\right)$. Applying Theorem 12 again, $\partial(f+g)$ is strongly metrically subregular at $\bar{x}$ for $\bar{x}^{*}+\bar{y}^{*}$, which completes the proof.

Next we investigate relationships between the strong metric subregularity and contingent derivative which is a graphical concept of derivative for set-valued maps and was introduced by Aubin in [13].

Definition 14. Let $F: X \rightrightarrows Y$ and $(\bar{x}, \bar{y}) \in \operatorname{gph} T$. The contingent derivative $\operatorname{DF}(\bar{x} \mid \bar{y})$ of $F$ at $(\bar{x}, \bar{y})$ is a set-valued mapping defined as

$$
D F(\bar{x} \mid \bar{y})(w):=\left\{z \in Y \mid(w, z) \in T_{\mathrm{gph} F}(\bar{x} \mid \bar{y})\right\} .
$$

Note that $\operatorname{D\partial f}(\bar{x} \mid \nabla f(\bar{x}))(w)=\left\{\nabla^{2} f(\bar{x}) w\right\}$ when $f$ is twice (Fréchet) differentiable (see, e.g., [14, Proposition 5.1.2]). For more details on the contingent derivative, one can refer to the comprehensive monograph [14] by Aubin and Frankowska.

Theorem 15. Given $f: X \rightarrow \overline{\mathbb{R}}$ and a pair $\left(\bar{x}, \bar{x}^{*}\right) \in \operatorname{gph} \partial f$, let $f$ be prox-regular at $\bar{x}$ for $\bar{x}^{*}$ with $r$ and subdifferentially continuous at $\bar{x}$ for $\bar{x}^{*}$. Consider the following two statements:

(i) the subdifferential $\partial f$ is strongly metrically subregular at $\left(\bar{x}, \bar{x}^{*}\right)$ with modulus $\kappa$ satisfying $\kappa^{-1}>r$, 
(ii) there is a constant $c>0$ such that $\operatorname{D\partial f}\left(\bar{x} \mid \bar{x}^{*}\right)$ is positive-definite with modulus $c$ in the sense that

$$
\left\langle z^{*}, w\right\rangle \geq c\|w\|^{2}, \quad \forall w \in X, z^{*} \in \operatorname{D\partial f}\left(\bar{x} \mid \bar{x}^{*}\right) .
$$

Then, implication $(i) \Rightarrow$ (ii) holds. Furthermore, the converse implication (ii) $\Rightarrow$ (i) also holds if in addition $\operatorname{dim} X<\infty$.

Proof. The proof is similar to [4, Corollary 3.7].

\section{Conflict of Interests}

The authors declare that there is no conflict of interests regarding the publication of this paper.

\section{Acknowledgments}

The authors would like to thank Ewa Bednarczuk for her helpful discussions on improving the quality of the paper. The research of J. J. Wang was partly supported by the Foundation of Heilongjiang Provincial Educational Department (12521147). The research of W. Song was supported in part by the National Natural Sciences Grant (no. 11371116).

\section{References}

[1] F. J. Aragón and M. H. Geoffroy, "Characterization of metric regularity of subdifferentials," Journal of Convex Analysis, vol. 15, no. 2, pp. 365-380, 2008.

[2] D. Drusvyatskiy and A. S. Lewis, "Tilt stability, uniform quadratic growth, and strong metric regularity of the subdifferential," SIAM Journal on Optimization, vol. 23, no. 1, pp. 256267, 2013.

[3] B. S. Mordukhovich and T. T. A. Nghia, "Second-order variational analysis and characterizations of tilt-stable optimal solutions in infinite-dimensional spaces," Nonlinear Analysis: Theory, Methods and Applications, vol. 86, pp. 159-180, 2013.

[4] F. J. Aragón and M. H. Geoffroy, "Metric subregularity of the convex subdifferential in Banach spaces," Journal of Nonlinear and Convex Analysis, vol. 15, no. 1, pp. 35-47, 2014.

[5] D. Drusvyatskiy, B. S. Mordukhovich, and T. T. A. Nghia, "Second-order growth, tilt stability and metric regularity of the subdifferential," Journal of Convex Analysis, vol. 21, no. 4, 2014.

[6] A. L. Dontchev and R. T. Rockafellar, Implicit Functions and Solution Map Pings: A View from Variational Analysis, Springer Monographs in Mathematics, Springer, Dordrecht, The Netherlands, 2009.

[7] A. D. Ioffe, "Metric regularity and subdifferential calculus," Russian Mathematical Surveys, vol. 55, pp. 501-558, 2000.

[8] B. S. Mordukhovich, Variational Analysis and Generalized Differentiation, I: Basic Theory, II: Applications, vol. 330, Springer, Berlin, Germany, 2006.

[9] R. A. Poliquin and R. T. Rockafellar, "Second-order nonsmooth analysis in nonlinear programming," in Recent Advances in Nonsmooth Optimization, D. Du, L. Qi, and R. Womersley, Eds., pp. 322-350, World Scientific Publishers, River Edge, NJ, USA, 1995.

[10] R. A. Poliquin and R. T. Rockafellar, "Generalized Hessian properties of regularized nonsmooth functions," SIAM Journal on Optimization, vol. 6, no. 4, pp. 1121-1137, 1996.
[11] R. A. Poliquin and R. T. Rockafellar, "Prox-regular functions in variational analysis," Transactions of the American Mathematical Society, vol. 348, no. 5, pp. 1805-1838, 1996.

[12] I. Ekeland, "On the variational principle," Journal of Mathematical Analysis and Applications, vol. 47, pp. 324-353, 1974.

[13] J. P. Aubin, "Contingent derivatives of set-valued maps and existence of solutions to nonlinear inclusions and differential inclusions," in Mathematical Analysis and Applications, Part A, vol. 7, pp. 159-229, Academic Press, New York, NY, USA, 1981.

[14] J. P. Aubin and H. Frankowska, Set-Valued Analysis, Systems \& Control: Foundations \& Applications, Birkhäuser, Boston, Mass, USA, 1990. 


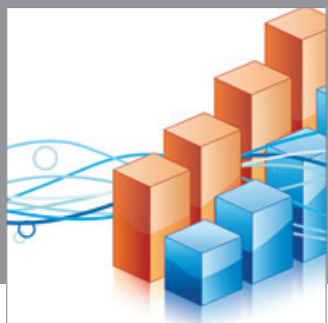

Advances in

Operations Research

mansans

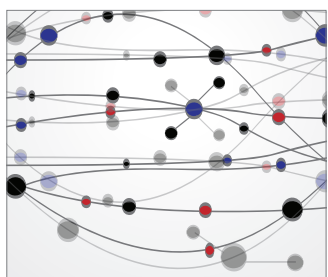

The Scientific World Journal
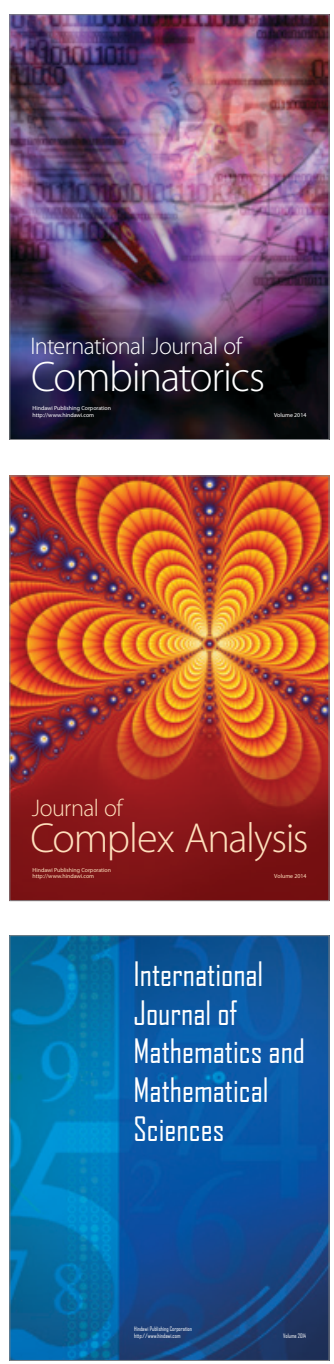
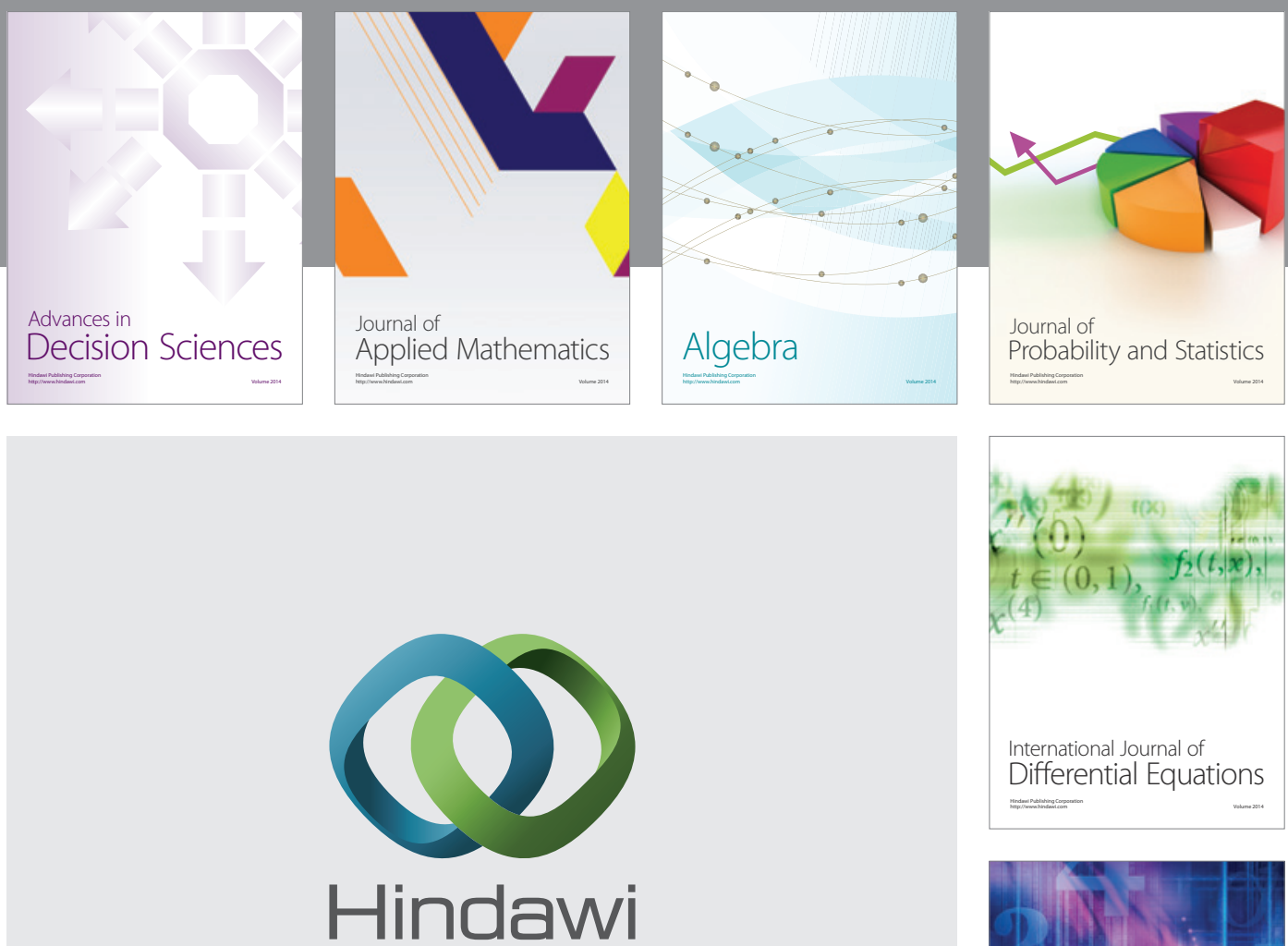

Submit your manuscripts at http://www.hindawi.com
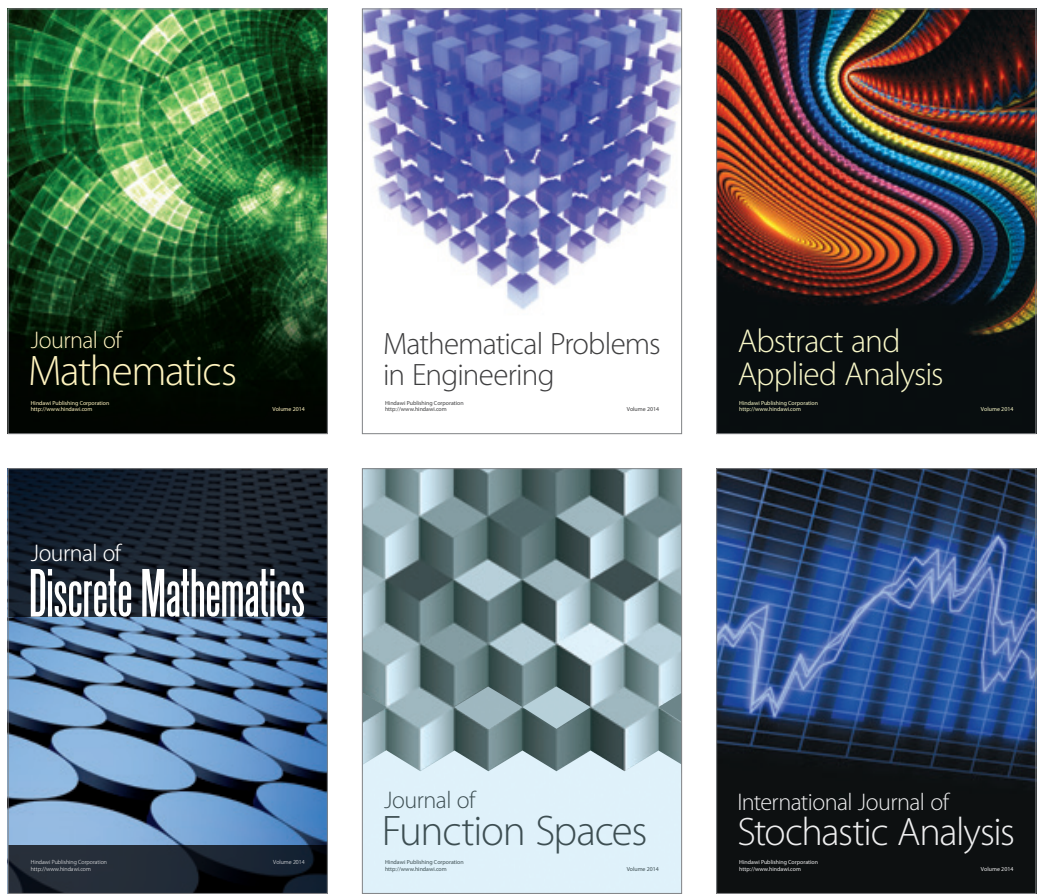

Journal of

Function Spaces

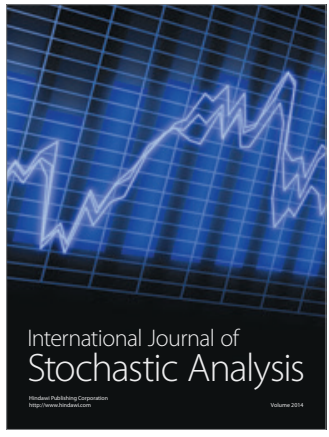

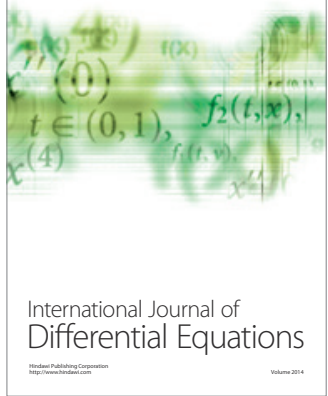
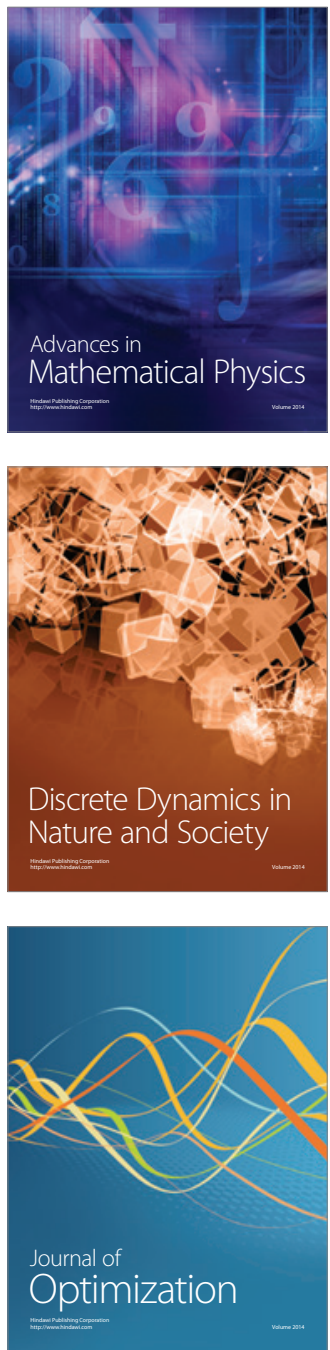